\title{
Can We Distinguish Chronic Myeloid Leukemia Presenting in the Lymphoblastic Phase from de novo Philadelphia- Positive Acute Lymphoid Leukemia?
}

\author{
Naoto Takahashi \\ Key words: Philadelphia chromosome, BCR-ABL, p190, p210, chronic myeloid leukemia, lymphoblastic \\ phase, acute lymphoid leukemia, lineage involvement, neutrophils-FISH
}

(Intern Med 49: 2535-2536, 2010)

(DOI: 10.2169/internalmedicine.49.4516)

The Philadelphia $(\mathrm{Ph})$ chromosome is a common cytogenetic abnormality in chronic myeloid leukemia (CML); it is also present in about $25 \%$ of adult patients with precursor B-lymphoblastic leukemia (ALL). Ph translocation results from fusion of $B C R$ at $22 \mathrm{q} 11.2$ with $A B L 1$ at $9 \mathrm{q} 34$, resulting in production of a BCR-ABL fusion protein. Two principal forms of the protein, p190 Kd and p210 Kd, arise from distinct breakpoints in the $B C R$ gene. In adults, about $50 \%$ of $\mathrm{Ph}+\mathrm{ALL}$ cases produce the p210 fusion protein, which is present in more than $95 \%$ of CML cases, and the remainder produce the p190 protein. CML invariably progresses to the blastic phase if it is left untreated, without a bone marrow transplantation or administration of a tyrosine kinase inhibitor. The blasts in the majority of patients in the blastic phase will be of the myeloid lineage; however, in approximately $20-30 \%$ of cases the blasts are lymphoblasts. Approximately $10 \%$ of patients in the blastic phase present without a chronic phase. In the absence of documented chronic phase CML, this lymphoblastic presentation poses a diagnostic challenge to differentiate the lymphoblastic phase CML from de novo Ph+ALL. The presence of p190 has been used to suggest de novo ALL rather than CML lymphoid crisis, but cases of CML with p190 have been reported.

In this journal [see Vol.49 (2010) pp 1297-1301], Sumimoto et al report two cases of $\mathrm{Ph}+$ leukemia in a paper entitled, "Blast phase of chronic myeloid leukemia presenting lymphoid phenotype with extremely short duration of chronic phase" (1). Both patients were treated with chemotherapy as de novo ALL. Even after chemotherapy reduced the numbers of peripheral leukemia cells by $90-95 \%$, the BCR-ABL fusion gene was found in almost all of the neutrophils as well as in the leukemia cells remaining in the peripheral blood. This suggests that multiple lineages arising from pluripotent stem cells are involved in $\mathrm{Ph}+$ leukemia, and that there is insidious lineage involvement of the $\mathrm{Ph}$ chromosome before acute presentation of leukemia. Although there was no evidence of a chronic phase during their clinical courses, these two cases could have been diagnosed as CML lymphoid crisis rather than de novo ALL, given the findings obtained with neutrophils-FISH (2). Moreover, these cases also could have been diagnosed as $\mathrm{Ph}+\mathrm{B}-\mathrm{ALL}$, based on the WHO classification, which defines $\mathrm{Ph}+\mathrm{ALL}$ as a "a neoplasm of lymphoblasts committed to the B-cell lineage in which the blasts harbor a translocation between the $B C R$ gene on chromosome 22 and the $A B L 1$ oncogene on chromosome 9." This suggests that a counterpart of de novo Ph+ALL should be a B-cell lineage progenitor cell. The WHO classification does not refer to lineage involvement of the $\mathrm{Ph}$ chromosome, except for the B-cell lineage.

Before the era of imatinib, it was generally thought that cases presenting in CML lymphoid crisis would have the characteristics of chronic phase CML once hematological remission was achieved. Jones et al compared the BCR-ABL transcript types (p190/p210), genotypes and phenotypes in 1855 cases with $\mathrm{Ph}+$ leukemia (3). As compared to p190 leukemia, p210 leukemia more frequently showed a CMLtype background, had higher blast-normalized BCR-ABL transcript levels, and more frequently showed a persistent BCR-ABL transcript in the absence of detectable lymphoblasts. This discrepancy between transcript level and hematological remission in p210 leukemia might indicate insidious multilineage involvement of the Ph chromosome before acute presentation of leukemia. CML has been thought to be a stem cell disease because not only is the $\mathrm{Ph}$ chromosome detected in myeloid cells, it is also detected in some 
lymphoid cells and in the patients' human hematopoietic stem cells (i.e., CD34+Thy1+ cells) (4). Recently, Tanizaki et al reported that CD34+CD38-CD19+ and CD34+CD38+ CD19+ cells from patients with CML blast crisis or $\mathrm{Ph}+$ ALL could self-renew and transfer the leukemia in NOD/ SCID mice. This finding suggests that leukemiarepopulating cells in transformed $\mathrm{Ph}+$ leukemia are mainly present in a B-cell lineage-committed fraction rather than among hematopoietic stem cells (5).

To date, clinical trials for $\mathrm{Ph}+\mathrm{ALL}$ have been performed without distinguishing among p190 de novo ALL, p210 de novo ALL, and CML presenting in lymphoidblastic phase. Might it be that there are differences in the response to chemotherapy and clinical outcomes among these diseases? If it proves clinically important to distinguish among them, it will be necessary to investigate an appropriate therapy for each disease in prospective clinical studies.

\section{References}

1. Sumimoto $H$, Tsujimura $H$, Ise $M$, et al. Blast phase of chronic myeloid leukemia presenting lymphoid phenotype with a chronic phase of extremely short duration. Intern Med 49: 1297-1301, 2010.

2. Takahashi N, Miura I, Kobayashi Y, et al. Fluorescence in situ hybridization monitoring of BCR-ABL-positive neutrophils in chronic-phase chronic myeloid leukemia patients during the primary stage of imatinib mesylate therapy. Int J Hematol 81: 235$241,2005$.

3. Jones D, Luthra R, Cortes $\mathrm{J}$, et al. BCR-ABL fusion transcript types and levels and their interaction with secondary genetic changes in determining the phenotype of Philadelphia chromosome-positive leukemias. Blood 112: 5190-5192, 2008.

4. Takahashi N, Miura I, Saitoh K, Miura AB. Lineage involvement of stem cells bearing the Philadelphia chromosome in chronic myeloid leukemia in the chronic phase as shown by a combination of fluorescence-activated cell sorting and fluorescence in situ hybridization. Blood 92: 4758-4763, 1998.

5. Tanizaki R, Nomura Y, Miyata Y, et al. Irrespective of CD34 expression, lineage-committed cell fraction reconstitutes and reestablishes transformed Philadelphia chromosome-positive leukemia in NOD/SCID/IL-2R $\gamma \mathrm{c}^{-1}$ mice. Cancer Sci 101: 631-638, 2010.

(C) 2010 The Japanese Society of Internal Medicine

http://www.naika.or.jp/imindex.html 